\title{
Peripheral and spinalTRPAI channels contribute to formalin-induced long-lasting mechanical hypersensitivity
}

This article was published in the following Dove Press journal: Journal of Pain Research

\author{
Vladimir A Martínez-Rojas' \\ Guadalupe García' \\ Roxana Noriega-Navarro' \\ Crystell G Guzmán-Priego² \\ Jorge E Torres-López ${ }^{2,3}$ \\ Vinicio Granados-Soto 4 \\ Janet Murbartián' \\ 'Departamento de Farmacobiología, \\ Centro de Investigación y de Estudios \\ Avanzados (Cinvestav), Unidad Coapa, \\ Ciudad de México, ${ }^{2}$ Laboratorio \\ Mecanismos del Dolor, Centro de \\ Investigación, División Académica \\ de Ciencias de la Salud, Universidad \\ Juárez Autónoma de Tabasco, ${ }^{3} \mathrm{Hospital}$ \\ Regional de Alta Especialidad "Dr. \\ Juan Graham Casasús”, Villahermosa, \\ Tabasco, ${ }^{4}$ Neurobiology of Pain \\ Laboratory, Departamento de \\ Farmacobiología, Cinvestav, Unidad \\ Coapa, Ciudad de México, México
}

Correspondence: Janet Murbartián Departamento de Farmacobiología, Cinvestav, Unidad Coapa, Calzada de los Tenorios 235, Colonia Granjas Coapa, 14330 Ciudad de México, México Email jmurbartian@gmail.com
Background: Transient receptor potential ankyrin 1 (TRPA1) is a non-selective cation channel expressed by a subset of nociceptive neurons that acts as a multimodal receptor. Its activity contributes to modulate nociceptive transmission in acute inflammatory pain. However, the role of this channel in chronic pain has been less studied. The purpose of this study was to investigate the local peripheral and spinal participation of TRPA1 channels in formalin-induced long-lasting hypersensitivity. Materials and methods: Formalin (1\%)-induced chronic hypersensitivity was determined by the application of von Frey filaments to ipsilateral and contralateral paws and through pharmacological testing using a selective TRPA1 blocker (A-967079). TRPA1 expression in the dorsal root ganglion (DRG) and spinal cord was analyzed by Western blotting.

Results: Formalin (1\%) injection produced acute flinching behavior $(1 \mathrm{~h})$ as well as secondary allodynia and hyperalgesia (12 days). Local peripheral pretreatment (10 min before) or posttreatment (6 days later) with A-967079 $(1-100 \mu \mathrm{M})$ partially prevented and reversed, respectively, in a dose-dependent manner, long-lasting secondary mechanical allodynia and hyperalgesia evoked by $1 \%$ formalin. Likewise, intrathecal pretreatment or posttreatment with A-967079 partially prevented and reversed, respectively, formalin-induced long-lasting hypersensitivity. A-967079 $(100 \mu \mathrm{M})$ completely abolished the pro-nociceptive effect of formalin (adjusted to $\mathrm{pH} 7.4$ ). Finally, formalin injection increased TRPA1 protein expression in the DRG and spinal cord.

Conclusion: Results indicate that TRPA1 expressed in the DRG and spinal cord plays a relevant role in formalin-induced long-lasting secondary nociceptive hypersensitivity.

Keywords: allodynia, chronic pain, formalin, hyperalgesia, TRPA1

\section{Introduction}

The transient receptor potential ankyrin 1 (TRPA1) is a polymodal cation channel that is highly expressed in nociceptors, where it functions to detect several pro-nociceptive molecules involved in pain and pruritus. ${ }^{1-4}$ However, TRPA 1 is also expressed in nonneuronal cells such as keratinocytes, ${ }^{5}$ megakaryocytes, ${ }^{6}$ or enterochromaffin cells. ${ }^{7}$ With regard to pain, this channel has been found in the dorsal root ganglion (DRG), trigeminal ganglion, and spinal cord. ${ }^{1,8-13}$ Activation of TRPA1 leads to activation of sensory neurons, release of inflammatory neuropeptides, and pain hypersensitivity. ${ }^{4,14}$

Several studies have reported that TRPA1 participates in inflammatory ${ }^{15-23}$ and neuropathic ${ }^{16,24-28}$ pain models. It has been reported that the TRPA1 channel blocker Chembridge-5861526 (CHEM), given intrathecally, diminishes formalin-induced secondary mechanical hypersensitivity ${ }^{18}$ suggesting that TRPA1 channels play an important role in the acute and short-lasting effects of formalin. The role of this channel 
in the development of long-lasting secondary mechanical allodynia and hyperalgesia induced by formalin remains unknown. Accordingly, we hypothesized that local peripheral and spinal TRPA1 underlies formalin-induced acute nociception and long-lasting secondary hyperalgesia and allodynia. Therefore, the purpose of this study was to investigate the participation of peripheral and spinal TRPA1 channels in the development and maintenance of formalin-induced acute nociception and long-lasting secondary mechanical allodynia and hyperalgesia in rats. Moreover, we analyzed TRPA1 protein expression in sites relevant to the nociceptive processing (DRG and spinal cord) in naïve and formalin-treated rats.

\section{Materials and methods Subjects}

Experiments were carried out in female Wistar rats (180$200 \mathrm{~g}$ ) of 8 to 10 weeks old at the beginning of experiments. At the time of the experiment, animals were 1 week old or older. Animals were housed in a room on a 12-h light/dark cycle and had free access to food and drinking water before the experiments. Experiments were conducted in accordance with the Guidelines of Ethical Standards for Investigation of Experimental Pain in Animals. ${ }^{29}$ In addition, the Institutional Animal Care and Use Committee approved our study (Cinvestav, Protocol 0092-14). All tests were undertaken during the light phase. The number of experimental animals was kept to a minimum.

\section{Reagents}

Formalin (Sigma-Aldrich, St. Louis, MO, USA) was freshly prepared and dissolved in $0.9 \% \mathrm{NaCl}$ (sterile saline) in most of the experiments. In one experiment, formalin was dissolved in phosphate-buffered saline ( $\mathrm{pH}$ 7.4). Then, (1E,3E)-1-(4-Fluorophenyl)-2-methyl-1-penten-3-one oxime (A-967079, Tocris Bioscience, Ellisville, MO, USA) was dissolved in dimethyl sulfoxide (DMSO) to make a stock solution and kept at $-20^{\circ} \mathrm{C}$ until the day of the experiment. The final concentration of DMSO was less than $1 \%(\mathrm{v} / \mathrm{v})$. For local peripheral administration, drugs were given in a volume of $50 \mu \mathrm{L}$ into the formalin-treated paw. For intrathecal (i.t.) administration, drugs were administered in a volume of $10 \mu \mathrm{L}$ (into the subarachnoid space) through lumbar puncture.

\section{Induction and assessment of acute nociception and long-lasting secondary mechanical allodynia and hyperalgesia}

Rats ( $n=6$ per group) were briefly immobilized in a manner that allowed access to the right hind limb. Then, animals received a subcutaneous injection of $1 \%$ formalin $(50 \mu \mathrm{L})$ into the dorsal surface on the right hind paw (ipsilateral) with a 30-gauge needle. Acute nociception was assessed using the formalin test. ${ }^{30}$ Rats were placed in open acrylic observation chambers for $30 \mathrm{~min}$ to allow them to acclimate to their surroundings; then, they were removed for formalin administration. Mirrors were placed in each chamber to enable unhindered observation. Nociceptive behavior was quantified as the number of flinches of the injected paw during 1 -min periods every $5 \mathrm{~min}$, for up to $60 \mathrm{~min}$ after injection. ${ }^{30}$ Flinching was characterized as rapid and brief withdrawal, or as flexing of the injected paw. Formalin-induced flinching behavior was biphasic. The initial acute phase (Phase 1,0-10 min) was followed by a relatively short quiescent period, which was then followed by a prolonged tonic response (Phase 2, 15-60 min). After acute nociceptive evaluation, animals were maintained for the next 12 days.

The hypersensitivity induced by formalin was tested at baseline and also 1, 3, 6, and 12 days after injection. However, for subsequent experiments, the sixth day was chosen to evaluate nociceptive behaviors as, at this time, nociceptive behaviors are already established. ${ }^{31,32}$ To evaluate the evoked nociceptive behaviors, rats were placed into testing cages on a wire-mesh bottom and allowed to acclimate for 30 min. Rats were stimulated 10 times with two von Frey filaments (Stoelting Co, Wood Dale, IL, USA) to the base of the third toe on the plantar surface of both paws. Three trials were carried out to determine the paw response frequency. A force of $10 \mathrm{mN}$ ( $1 \mathrm{~g}$ ) does not activate cutaneous nociceptors in naïve rats, as it does not lead to paw withdrawal. Thus, a response to this filament is an indication of allodynia. Likewise, a force of $250 \mathrm{mN}(26 \mathrm{~g})$ is considered a noxious stimulus. ${ }^{33}$ An increased response to this filament indicates hyperalgesia. Rats were killed in a $\mathrm{CO}_{2}$ chamber at the end of the experiment.

\section{Western blot analysis}

Western blot analysis was used to determine the expression of TRPA1 in ipsilateral DRG and the dorsal spinal cord. For this, naïve and formalin-treated rats ( $n=3$ per group) were killed by decapitation. DRGs (L4-L6), corresponding to the afferent pathway from the hind paw, and the dorsal spinal cord (L4-L6 segment) were carefully removed and processed as previously described. ${ }^{34}$ Tissues from individual animals were homogenized in ice-cold lysis buffer (in mM: $150 \mathrm{NaCl}, 50$ Tris- $\mathrm{HCl}, 5$ EDTA), pH 7.4 for $30 \mathrm{~min}$ at $4{ }^{\circ} \mathrm{C}$. The protease inhibitors phenylmethylsulfonyl fluoride $(1 \mathrm{mM})$, aprotinin $(10 \mu \mathrm{g} / \mathrm{mL})$, leupeptin $(10 \mu \mathrm{g} / \mathrm{mL})$, pepstatin A $(10 \mu \mathrm{g} / \mathrm{mL})$, 
and the surfactant $0.1 \%$ Triton X-100 (Sigma-Aldrich, St. Louis, MO, USA) were added to the lysis buffer immediately prior to use. The homogenate was then centrifuged (Eppendorf, Hamburg) at 14,000 rpm for $10 \mathrm{~min}$ to remove cellular debris. The resultant supernatant was used to measure protein concentration by using Bradford's method (Bio-Rad, Hercules, CA, USA).

Fifty micrograms of total protein were separated by electrophoresis in 10\% SDS-polyacrylamide gels and transferred to polyvinylidene fluoride membranes. Membranes were blocked in 5\% non-fat milk in PBS at $\mathrm{pH} 7.4$ (in mM: $137 \mathrm{NaCl}, 2.7 \mathrm{KCl}, 10 \mathrm{Na}_{2} \mathrm{HPO}_{4}$, and $2 \mathrm{KH}_{2} \mathrm{PO}_{4}$ ) with $0.05 \%$ Tween-20 for $1 \mathrm{~h}$. After that, they were washed and incubated overnight at $4{ }^{\circ} \mathrm{C}$ in $5 \%$ non-fat dry milk/PBS containing rabbit anti-TRPA1 antibody $(1: 2,000$, Cat. No. 68847, Abcam, Cambridge, MA, USA). Membranes were incubated for $1 \mathrm{~h}$ at room temperature in $1 \%$ non-fat milk/ PBS containing the horseradish peroxidase-conjugated secondary antibody (bovine anti-rabbit 1:3000, Cat. No. sc-2370, Santa Cruz Biotechnology, Dallas, TX, USA or goat anti-mouse 1:10,000, Cat. No. 115-035-003, Jackson Immuno Research, West Grove, PA, USA). Protein signal detection was achieved with the ECL chemiluminescence system (Millipore, Billerica, MA, USA). The next day, blots were incubated with a monoclonal antibody directed against $\beta$-actin (1:5,000, Cat. No. MAB1501R, Millipore, Billerica, MA, USA), which was used as internal control to normalize TRPA1 protein expression level. Bands were analyzed with Image Studio Ver. 4.0 (LI-COR, Lincoln, NE, USA).

\section{Experimental design}

To determine whether blockade of TRPA1 channels at the peripheral or spinal level modifies formalin-induced secondary allodynia and hyperalgesia, we used the highly selective TRPA1 blocker A-967079 $\left(\mathrm{IC}_{50}: 289 \mathrm{nM}\right) .{ }^{24}$ Dose selection was based on previous studies ${ }^{17,24}$ and pilot experiments in our laboratory. Formalin (1\%) was selected because, at this concentration, formalin activates $\mathrm{C}$ fibers wherein TRPA1 is mainly expressed. . $^{3,35}$

In order to assess the participation of local peripheral and spinal TRPA1 channels in the development of formalininduced secondary allodynia and hyperalgesia, rats received a subcutaneous (s.c.) injection into the dorsal surface of the right hind paw $(50 \mu \mathrm{L})$ or an i.t. injection $(10 \mu \mathrm{L})$ of vehicle (1\% DMSO) or increasing doses of A-967079 (1-100 $\mu \mathrm{M}) 10$ min before $1 \%$ formalin injection into the right (ipsilateral) paw. Allodynia and hyperalgesia were evaluated 6 days later in both paws. To determine whether A-967079 acted locally, the maximal dose of A-967079 used $(100 \mu \mathrm{M})$ was injected into the left (contralateral) paw $10 \mathrm{~min}$ before a $1 \%$ formalin injection into the right paw, and the effect on nociceptive behavior was assessed in both paws.

In order to determine the participation of local peripheral and spinal TRPA1 channels in the maintenance of formalininduced secondary allodynia and hyperalgesia, rats received a s.c. injection into the dorsal surface of the right hind paw $(50 \mu \mathrm{L})$ or an i.t. injection $(10 \mu \mathrm{L})$ of vehicle $(1 \%$ DMSO $)$ or increasing doses of A-967079 $(1-100 \mu \mathrm{M}) 6$ days after 1\% formalin injection into the right (ipsilateral) paw. Animals were tested before drug administration to register a baseline of nociceptive behaviors. Allodynia and hyperalgesia were evaluated $1 \mathrm{~h}$ after drug administration because, at this time, we observed the maximal antinociceptive response.

In order to discharge the effects of hydrogen ions $\left(\mathrm{H}^{+}\right)$in the development of formalin-induced secondary allodynia and hyperalgesia, rats received a local peripheral pretreatment with vehicle (1\% DMSO) or A-967079 $(100 \mu \mathrm{M}) 10 \mathrm{~min}$ before the administration of $1 \%$ formalin $\mathrm{pH} 7.4$, instead of regular formalin (without controlling the $\mathrm{pH}$ ).

In order to assess the role of TRPA1 channels in sites related to nociceptive processing induced by formalin, we determined the expression of this channel at the ipsilateral DRG and dorsal spinal cord at $1 \mathrm{~h}$ as well as on days 1,6 , and 12 after the $1 \%$ formalin injection.

\section{Data and statistical analysis}

Behavioral data are expressed as mean \pm SEM of the hind paw withdrawal response of six rats for each group. Protein expression data are the mean \pm SEM of three independent experiments.

Data were analyzed by one-way analysis of variance (ANOVA) followed by the Student-Newman-Keuls post hoc test; $p$-values less than 0.05 were considered significant. Statistical analyses were conducted using GraphPad Prism 6.0 (GraphPad Software, Inc., La Jolla, CA, USA).

\section{Results}

\section{Acute and secondary allodynia and hyperalgesia induced by formalin}

Formalin administration into the dorsal surface of the right hind paw resulted in acute nociception (spontaneous flinching) lasting approximately $1 \mathrm{~h}$ (data not shown) as well as secondary mechanical allodynia and hyperalgesia lasting from 1 to 12 days in the injected (ipsilateral; Figure 1A and C) and in the uninjured (contralateral; Figure 1B and D) hind paw. This was observed as a bilateral increase in the paw withdrawal responses to application of von Frey filaments, which was 
A

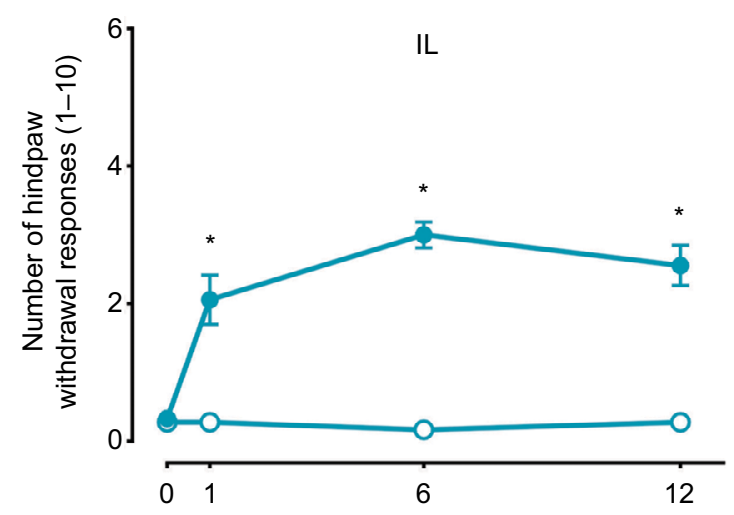

C

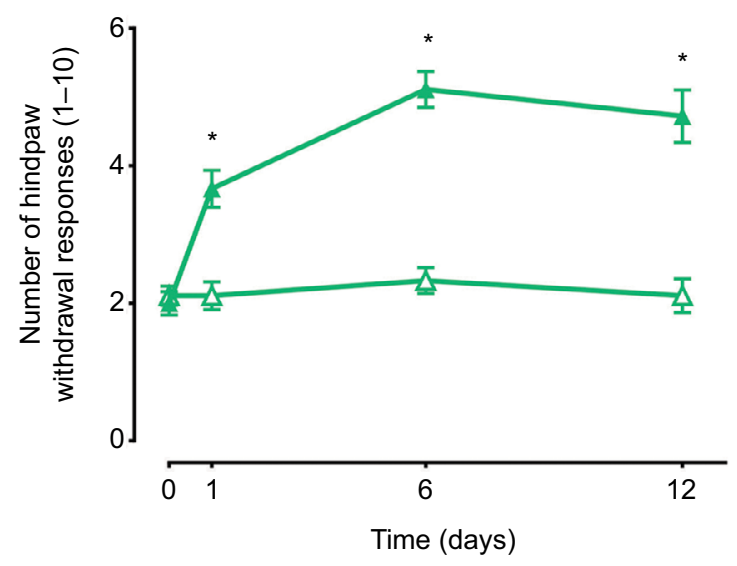

B

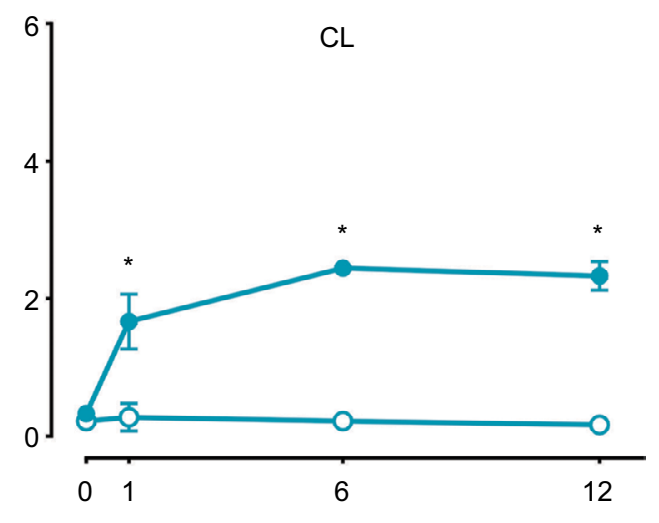

D

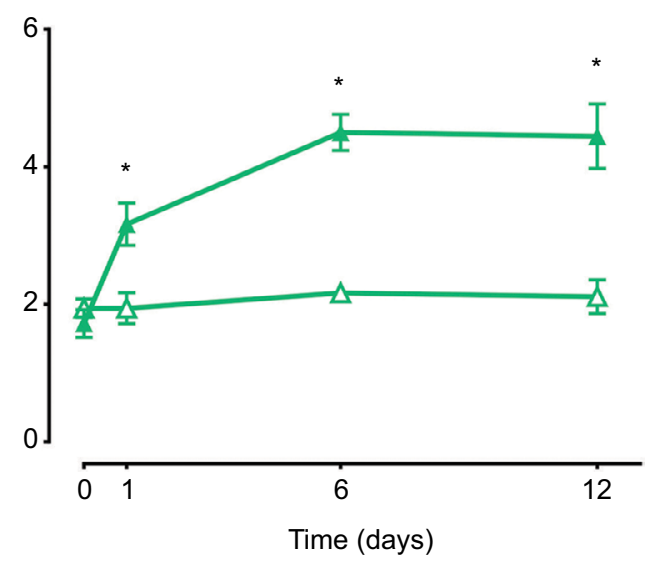

Figure I The time course of the mean number of paw withdrawal responses ( $n=6 \pm$ SEM) to 10 applications of von Frey filaments of two different bending forces 10 (circles, $\mathbf{A}$ and $\mathbf{B}$ ) and $250 \mathrm{mN}$ (triangles, $\mathbf{C}$ and $\mathbf{D})$ following subcutaneous administration of $1 \%$ formalin (I\% F).

Note: $p<0.05$ by two-way ANOVA followed by the Student-Newman-Keuls test.

Abbreviations: IL, ipsilateral; CL, contralateral; ANOVA, analysis of variance.

significant 1 day after formalin administration and lasted for at least 12 days $(p<0.05)$. For experiments with the TRPA 1 channel blocker, the sixth day was chosen to evaluate nociceptive behaviors as previously reported. ${ }^{36}$ In addition, effects in the contralateral paw were omitted for the sake of clarity.

\section{Effects of A-967079 on formalin-induced acute and long-lasting hypersensitivity}

Local peripheral and i.t. pretreatment (10 min before) with A-967079 $(1-100 \mu \mathrm{M})$ partially prevented formalin-induced acute $(1 \mathrm{~h})$ nociceptive flinching during phases 1 and 2 $(p<0.05$, Figure 2). Moreover, local peripheral $(p<0.05$, Figure 3$)$ or spinal $(p<0.05$, Figure 4$)$ pre- or posttreatment (6 days later) with A-967079 $(1-100 \mu \mathrm{M})$ partially prevented and reversed, respectively, $1 \%$ formalin-induced secondary mechanical allodynia and hyperalgesia in ipsilateral and contralateral paws. Of note, local peripheral administration of A-967079 $(100 \mu \mathrm{M})$ into the contralateral paw did not affect formalin-induced hypersensitivity, indicating that the antinociceptive effect of A-967079 is mediated by the blockade of TRPA1 located in the periphery (Figure 3).

Formalin (adjusted to $\mathrm{pH}$ 7.4) injection produced lesser nociceptive behaviors than formalin dissolved in saline. Interestingly, local peripheral pretreatment with A-967079 $(100 \mu \mathrm{M})$ completely prevented formalin ( $\mathrm{pH} 7.4)$-induced long-lasting allodynia and hyperalgesia $(p<0.05$, Figure 5$)$.

\section{Effect of formalin on TRPAI expression in DRG and spinal cord}

Western blot analysis identified a $123-\mathrm{kDa}$ band corresponding to the molecular weight expected for TRPA1 in the ipsilateral DRG and dorsal spinal cord of naïve and formalin-treated rats (Figure 6). Moreover, formalin (1\%) injection increased TRPA 1 protein expression on days 6 and 1 after injection in the ipsilateral DRG $(p<0.05$, Figure $6 \mathrm{~A})$ and dorsal spinal cord $(p<0.05$, Figure $6 \mathrm{~B})$. No change in TRPA1 protein expression was detected in the contralateral DRG or dorsal horn (data not shown). 
A

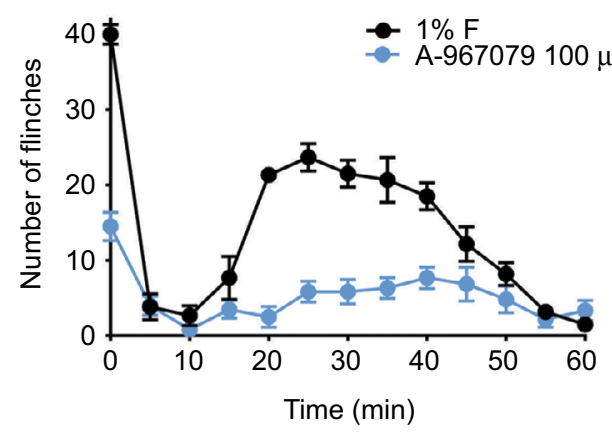

C

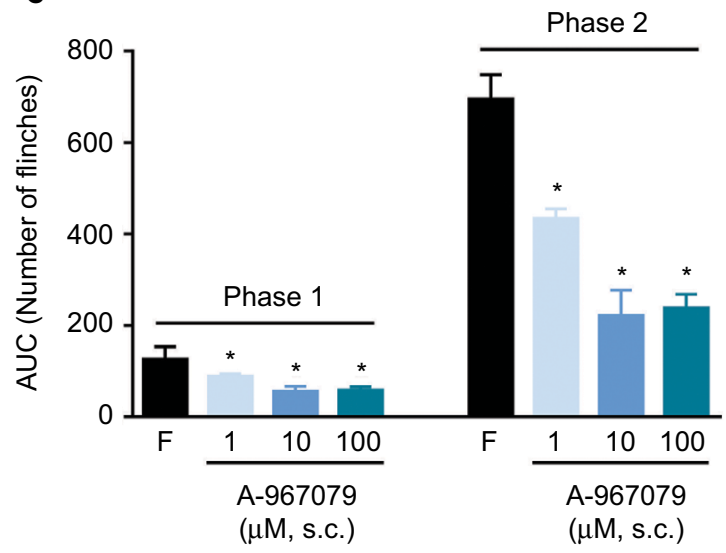

\section{B}

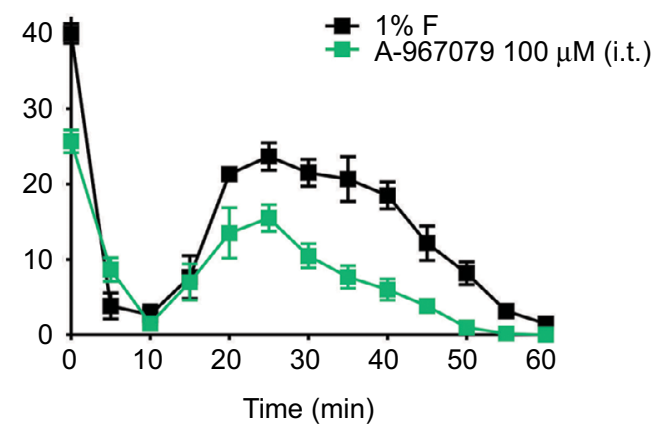

D

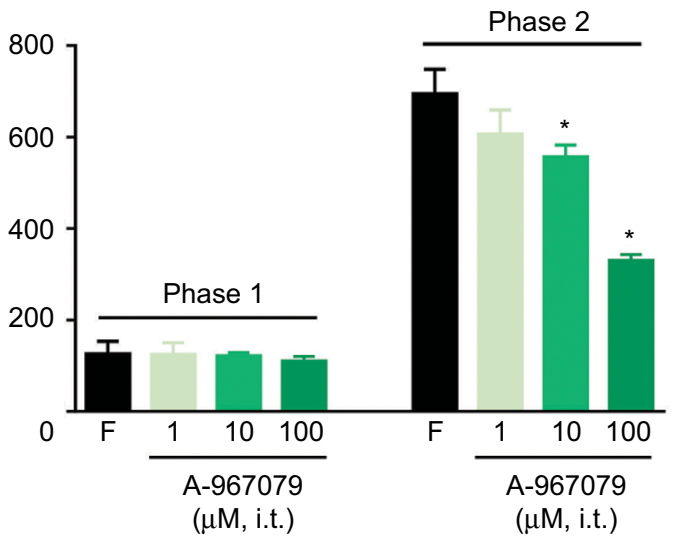

Figure 2 Antinociceptive effect of the local peripheral (s.c.; A and C) and intrathecal (i.t.; B and D) pretreatment with A-967079 (I-I00 $\mu$ M) in rats subjected to the I\% formalin test.

Notes: (A and B) The time course observed during phases I and 2 of the formalin test after administration of $100 \mu M$ of A-967079 into the dorsal part of the paw (A) or i.t. (B) 10 min before formalin injection. $\mathbf{C}$ and $\mathbf{D}$ show the data on the area under the curve for the phases I and 2 for local peripheral and intrathecal administration, respectively. Data are mean ( $n=6 \pm$ SEM). In plots $\mathbf{C}$ and $\mathbf{D}$, ${ }^{*} p<0.05$, significantly different from the formalin (F) group, by one-way ANOVA followed by the Student-Newman-Keuls test. Abbreviation: ANOVA, analysis of variance.

\section{Discussion}

In this study, we found that either local peripheral or i.t. administration of the TRPA1 channel blocker A-967079 prevented acute nociception in the formalin test. Furthermore, this drug prevented and reversed formalin-induced long-lasting hypersensitivity in rats. As formalin is a TRPA1 channel agonist ${ }^{35}$ and A-967079 is a highly selective TRPA1 channel blocker, ${ }^{24}$ our data suggest that peripheral and spinal TRPA1 channels participate in acute nociception as well as in the development and maintenance of long-lasting secondary allodynia and hyperalgesia induced by formalin. These data suggest that acute nociception and secondary allodynia and hyperalgesia depend on TRPA1 channel-induced persistent small afferent input as well as a sensitized dorsal horn. Our results agree with previous observations demonstrating that TRPA1, indeed, participate in formalin-induced acute nociception. ${ }^{18,35}$ Moreover, the fact that acute nociception induced by the selective TRPA1-channel agonist allyl isothiocyanate (AITC) is prevented by A-967079 further supports that TRPA1 plays an important role in formalin-induced acute nociception. ${ }^{24}$ However, to our knowledge, this is the first report about the anti-allodynic and anti-hyperalgesic effect of A-967079 in formalin-induced long-lasting nociceptive hypersensitivity. Our study agrees with a previous report showing that the TRPA1-channel blocker CHEM diminishes formalin-induced secondary mechanical hypersensitivity $(24 \mathrm{~h}){ }^{18}$ suggesting that TRPA1 channels play an important role in the short- (1 day) and long-lasting (6-12 days) effects of formalin. Moreover, our data concord with those showing that i.t. injection of the TRPA1-channel blocker HC-030031 reduces long-lasting (28 days) mechanical and cold allodynia induced by complete Freund's adjuvant (CFA) in mice. ${ }^{11}$ The fact that A-967079 blocks AITC-induced acute nociceptive behaviors in vivo, ${ }^{24}$ further suggest that TRPA1 channels are important for the development and maintenance of nociceptive hypersensitivity induced by formalin. Moreover, our data agree with several studies reporting that other TRPA1 antagonists (HC030031, CHEM) or genetic deletion of Trpal reduces nociceptive hypersensitivity induced by colorectal distension, pancreatitis, colitis, osteoarthritis, REM-sleep deprivation, skin incision, intraplantar capsaicin, carrageenan, and peripheral nerve injury. ${ }^{15-24,28,37,38}$ Thus, our 


\section{Pre-T}

A

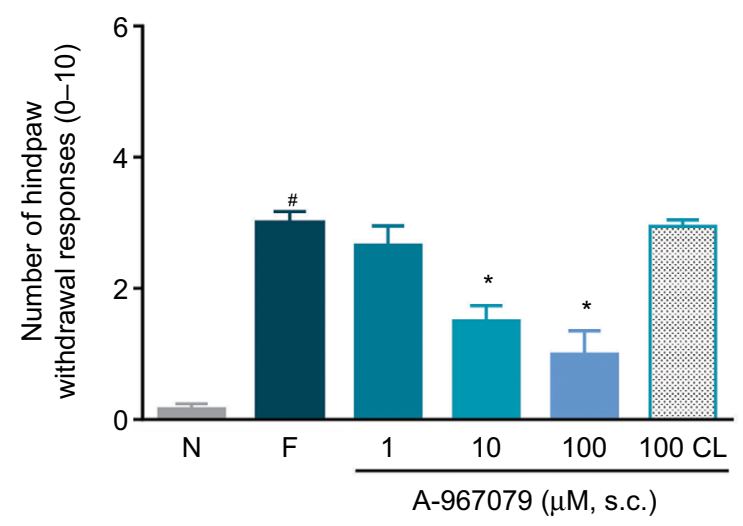

B

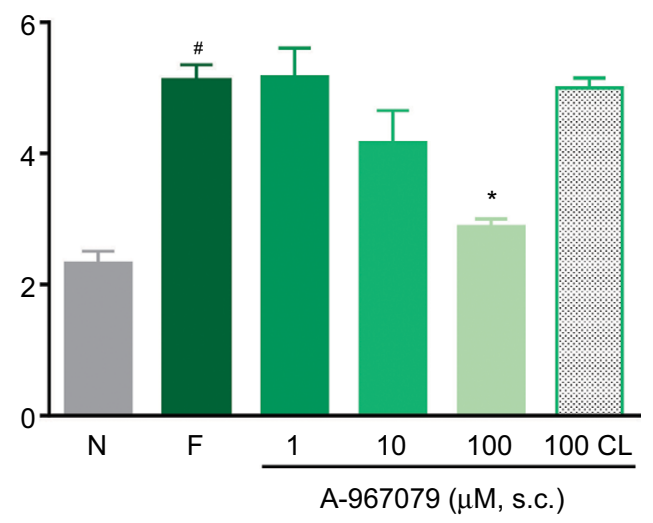

Post-T

C

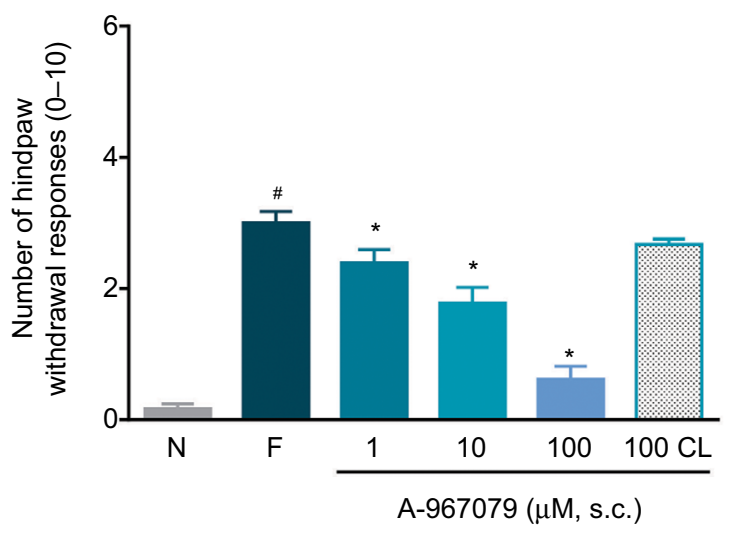

D

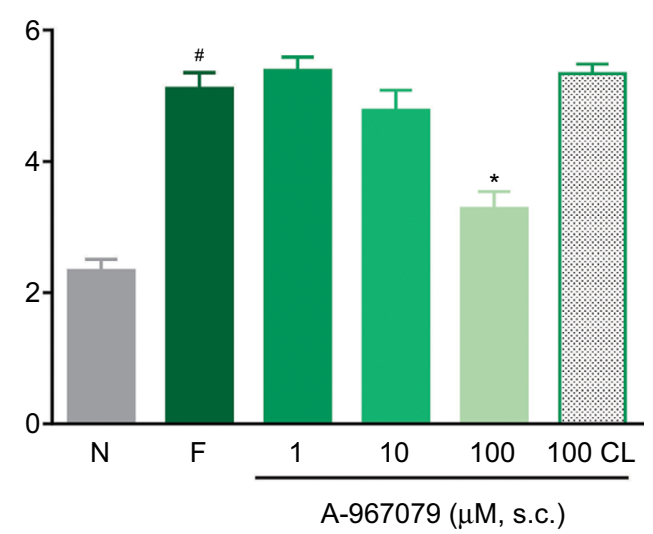

Figure 3 Antinociceptive effect of the local peripheral (s.c.) pretreatment (A and B) or posttreatment (C and D) with A-967079 (I-I00 $\mu$ M) on formalin-induced secondary mechanical allodynia ( $\mathbf{A}$ and $\mathbf{C}$ ) and hyperalgesia (B and $\mathbf{D})$ in rats.

Notes: Data are expressed as the mean ( $n=6 \pm$ SEM) paw withdrawal responses to the application of von Frey filaments ( 10 and $250 \mathrm{mN})$ to the plantar surface of rat paws before (naïve, $N$ ) and after $1 \%$ formalin (F). In all cases, ${ }^{*} p<0.05$ versus $N$ group; ${ }^{*} p<0.05$ versus $F$ group, by one-way ANOVA followed by the Student-Newman-Keuls test. Abbreviations: pre-T; pretreatment; post-T, posttreatment; ANOVA, analysis of variance; CL, contralateral.

results extend these observations by showing that blockade of TRPA1 channels has important consequences on the development and maintenance of formalin-induced nociceptive hypersensitivity. Some studies have reported that oral administration of A-967079 does not diminish mechanical allodynia induced by CFA, chronic constriction injury, or spinal nerve ligation models. ${ }^{24}$ It is likely that this discrepancy is due to the low amount of compound that reaches local peripheral or spinal sites after oral administration. In support of this, i.t. administration of CHEM or HC-030031 reduces tactile allodynia in neuropathic rats. ${ }^{26,27}$

It has been established that activation of TRPA1 channels by its agonists (cinnamaldehyde, AITC, and formalin) in the paw produces mechanical sensitization in rats. ${ }^{15,16,35,39}$ Forma- lin injection induces peripheral and central sensitization. In the first case, there is evidence that formalin injection leads to serotonin (5-HT), histamine, IL-1 $\beta$, and TNF $\alpha$ release at the periphery, which in turn activate their receptors to produce direct nociception or sensitization. ${ }^{40-42}$ With regard to central effects, formalin injection starts a cascade of events including release of glutamate, prostaglandins, and 5-HT at the dorsal horn spinal cord ${ }^{43,44}$ and activation of microglia which, in turn, releases nitric oxide, nerve growth factor, cytokines (IL-1 $\beta$ and TNF $\alpha$ ), and brain-derived neurotrophic factor (BDNF). ${ }^{31,45,46}$ Acting at their receptors, these molecules promote central sensitization and nociception. Thus, the local peripheral anti-allodynic and anti-hyperalgesic effects of A-967079 could be due, in the first instance, to the 
Pre-T

A

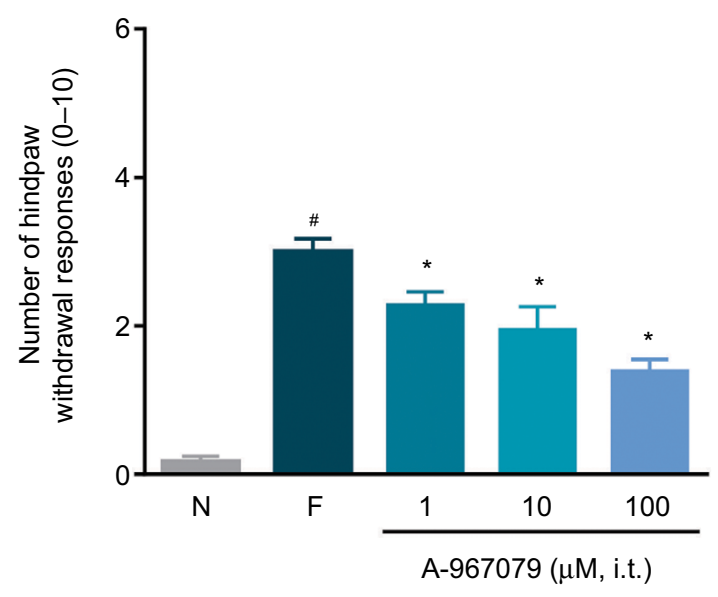

B

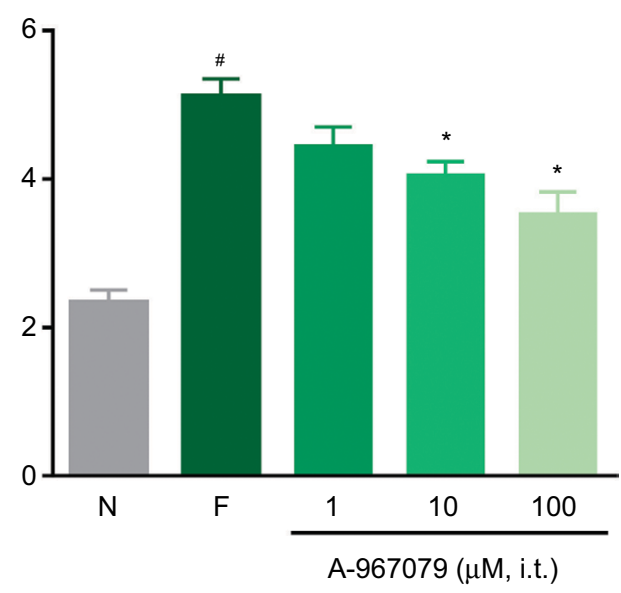

Post-T

C

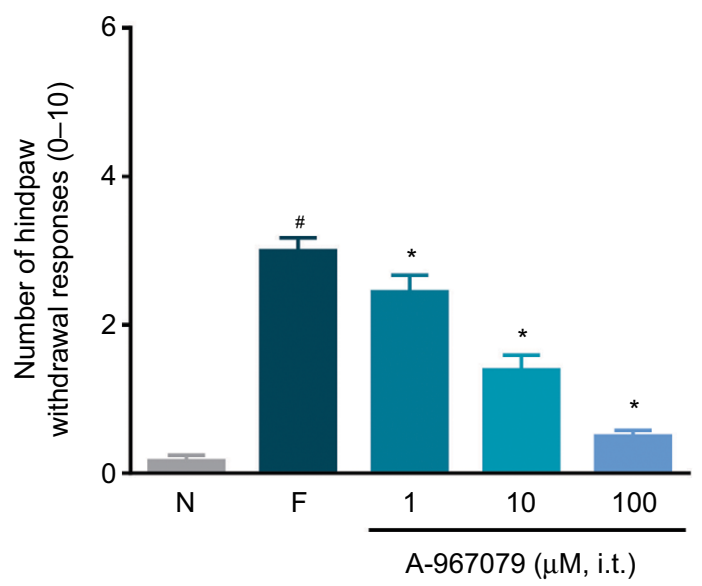

D

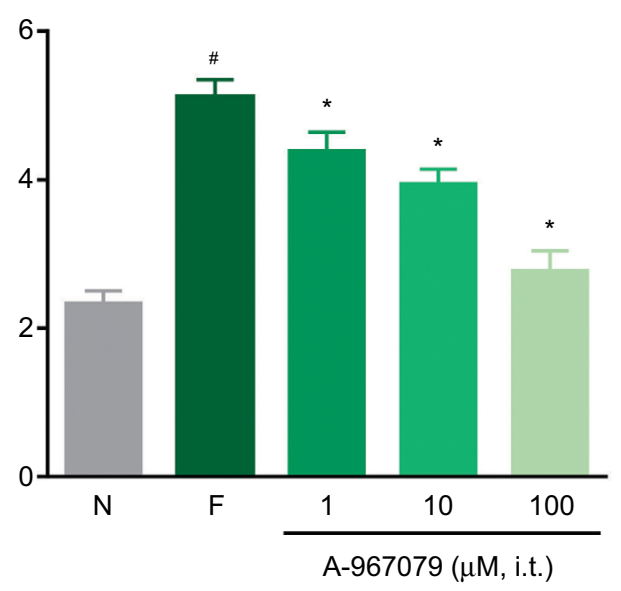

Figure 4 Antinociceptive effect of the intrathecal (i.t.) pretreatment (A and B) or posttreatment (C and D) with A-967079 (I-I00 $\mu$ M) on formalin-induced secondary mechanical allodynia (A and $\mathbf{C})$ and hyperalgesia $(\mathbf{B}$ and $\mathbf{D})$ in rats.

Notes: Data are expressed as the mean ( $n=6 \pm$ SEM) paw withdrawal responses to the application of von Frey filaments ( 10 and $250 \mathrm{mN})$ to the plantar surface of rat paws before (naïve, $N$ ) and after I\% formalin (F). In all cases ${ }^{*} p<0.05$ versus $N$ group; ${ }^{*} p<0.05$ versus $F$ group, by one-way ANOVA followed by the Student-Newman-Keuls test. Abbreviations: pre-T, pretreatment; post-T, posttreatment; ANOVA, analysis of variance.

blockade of TRPA1 channels located on peripheral endings of primary afferent neurons. This will reduce the release of several pro-nociceptive substances, avoiding peripheral sensitization. On the other hand, A-967079 - given into the spinal cord - may reduce formalin-induced secondary allodynia and hyperalgesia by diminishing release of pronociceptive molecules as well as activation of microglia. In addition, i.t. A-967079 could reduce dorsal root reflexes or direct antidromic activation and cutaneous blood flow leading to a reduction in substance $\mathrm{P}$ and calcitonin generelated peptide release in peripheral and central terminals of primary afferent neurons. ${ }^{24,25,47,48}$ Moreover, activation of spinal TRPA1 channels leads to an increase of the excitatory synaptic transmission in substantia gelatinosa neurons, ${ }^{49,50}$ which in turn reduces activation thresholds and enhances afferent activity. ${ }^{39}$ Thus, i.t. A-967079 could block this excitatory synaptic transmission and some consequences of the descending pain facilitation. ${ }^{18}$ All of these actions would reduce the pre- and postsynaptic excitability of afferent and convergent pain-relay neurons.

Local peripheral or i.t. administration of A-967079 produced a partial reversal of secondary allodynia and hyperalgesia in the formalin model. This result may suggest that other mechanisms, besides activation of TRPA1 chan- 
A

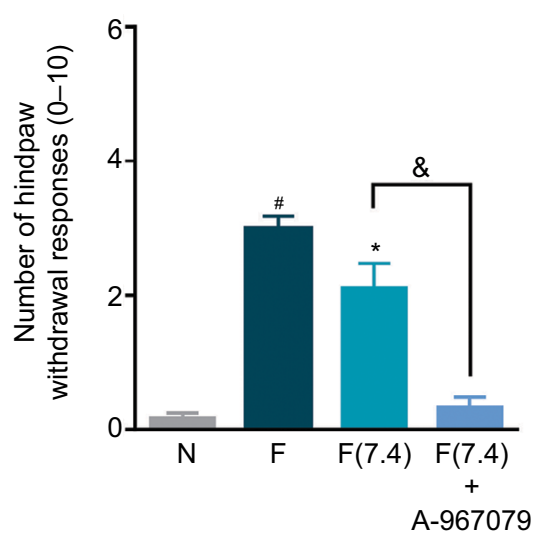

B

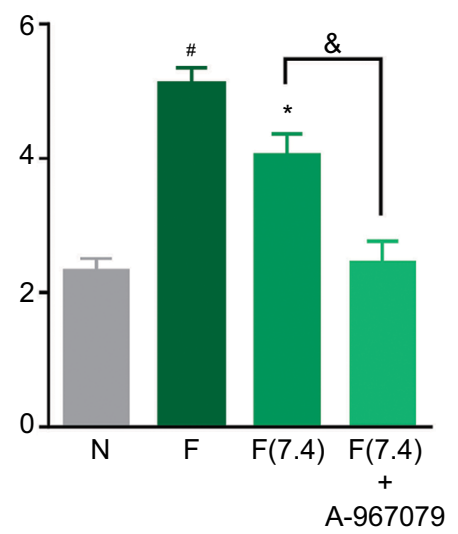

Figure 5 The antinociceptive effect of the local peripheral pretreatment with A-967079 $(100 \mu \mathrm{M})$ on $1 \%$ formalin $(\mathrm{pH} 7.4)$-induced secondary mechanical allodynia $(\mathbf{A})$ and hyperalgesia (B) in rats.

Notes: Data are expressed as the mean ( $\mathrm{n}=6 \pm$ SEM) paw withdrawal responses to the application of von Frey filaments ( 10 and $250 \mathrm{mN})$ to the plantar surface of rat paws before (naïve, N) and after $1 \%$ formalin without control of $\mathrm{pH}[\mathrm{F}]$ or $\mathrm{I} \%$ formalin at $\mathrm{pH} 7.4[\mathrm{~F}(7.4)]$. In all cases, ${ }^{*} p<0.05$ versus $\mathrm{N}$ group; ${ }^{*} p<0.05$ versus $\mathrm{F}$ group; ${ }^{\circledR} p<0.05$ versus $\mathrm{F}(7.4)$ group, by one-way ANOVA followed by the Student-Newman-Keuls test.

Abbreviation: ANOVA, analysis of variance.

A
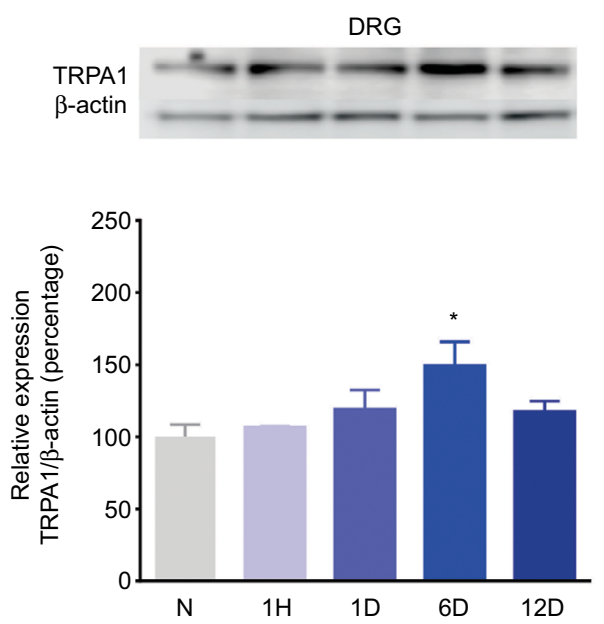

B
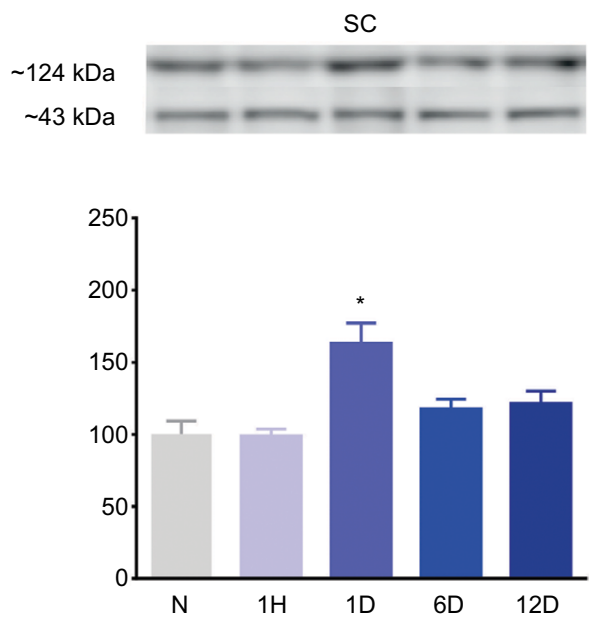

Figure 6 Effect of $1 \%$ formalin on the TRPAI protein expression levels in the ipsilateral DRGs (A) and dorsal SC (L4-L6) (B) from rats.

Notes: Insets show representative blots obtained with TRPAI and $\beta$-actin primary antibodies. The specific signal for TRPAI was quantified and plotted as the percentage of expression against $\beta$-actin ( $n=3 \pm$ SEM). ${ }^{*} p<0.05$ compared to the naïve group, by one-way ANOVA followed by the Student-Newman-Keuls test.

Abbreviations: N, naïve; IH, I hour; ID, I day; 6D, 6 days; I2D, I2 days; ANOVA, analysis of variance; DRG, dorsal root ganglion; SC, spinal cord.

nels, are involved in the development and maintenance of secondary allodynia and hyperalgesia. Former studies have established that activation of acid-sensing ion channels (ASICs) and TRPV1 is a common feature in inflammatory pain in which $\mathrm{pH}$ is not controlled. ${ }^{51,52}$ Furthermore, we have demonstrated that hydrogen ions $\left(\mathrm{H}^{+}\right)$indeed contribute to the development of secondary allodynia and hyperalgesia induced by formalin. ${ }^{53}$ Thus, injection of formalin without pH control would stimulate several targets, including ASICs, TRPV $1,{ }^{54}$ and TRPA $1{ }^{35}$ channels. In order to discharge the participation of $\mathrm{H}^{+}$and activation of TRPV1 and ASICs, we designed an experiment controlling the $\mathrm{pH}$ of formalin to 7.4 . We observed that $1 \%$ formalin at $\mathrm{pH} 7.4$ produced lower levels of secondary allodynia and hyperalgesia than $1 \%$ formalin (without $\mathrm{pH}$ control). This result agrees with a previous observation showing that $0.5 \%$ formalin at $\mathrm{pH}$ 7.4 induced lower nociceptive behaviors than either $0.5 \%$ formalin at $\mathrm{pH} 5.8$ or non-pH-adjusted formalin. ${ }^{53}$ In this condition ( $1 \%$ formalin at $\mathrm{pH} 7.4$ ), local peripheral administration of A-967079 completely abolished development of formalin-induced secondary allodynia and hyperalgesia in rats. Thus, it seems that under controlled conditions of $\mathrm{pH}$, the only target responsible of the nociceptive hypersensitivity is TRPA1 channel.

In line with the behavioral results, Western blot data revealed that formalin injection increases TRPA1 protein 
expression in the ipsilateral DRG and dorsal region of the spinal cord. Thus, our data indicate that this channel is located in sites related to the peripheral and spinal processing of pain. Our data are the first to demonstrate that formalin enhances TRPA1 protein expression in the DRG and spinal cord. These results concord with those from other studies demonstrating that CFA, colitis, and nerve injury enhances TRPA1 protein and mRNA expression in the DRG of rats and mice. ${ }^{1,8,11,39,55-58}$

\section{Conclusion}

Formalin produces long-lasting nociceptive hypersensitivity, which is sensitive to TRPA1 blockade. Data suggest that the peripheral and spinal TRPA1 channel located in the DRG and spinal cord is important for the development and maintenance of formalin-induced secondary allodynia and hyperalgesia. This channel could be a target to develop useful drugs to treat long-lasting nociceptive hypersensitivity.

\section{Acknowledgments}

This study is part of the Ph.D. dissertation of Vladimir A Martínez-Rojas. Vladimir A Martínez-Rojas (fellowship 261132) and Guadalupe García (fellowship 261810) are Conacyt fellows. Roxana Noriega-Navarro received a postdoctoral fellowship from Conacyt. Partially supported by UJAT (grant OP/PFCE-2016-27MSU0018V-07-01).

\section{Author contributions}

$\mathrm{JM}$ and VAM-R conceived, designed, and conducted the experiments. VAM-R, RN-N, CGG-P, and GG conducted the experiments. JM, JET-L, and VG-S supervised the experiments and analyzed data. All authors contributed toward data analysis, drafting, and critically revising the paper, gave final approval of the version to be published, and agreed to be accountable for all aspects of the work.

\section{Disclosure}

The authors report no conflicts of interest in this work.

\section{References}

1. Story GM, Peier AM, Reeve AJ, et al. ANKTM1, a TRP-like channel expressed in nociceptive neurons, is activated by cold temperatures. Cell. 2003;112(6):819-829.

2. Nagata K, Duggan A, Kumar G, García-Añoveros J. Nociceptor and hair cell transducer properties of TRPA1, a channel for pain and hearing. J Neurosci. 2005;25(16):4052-4061.

3. Bautista DM, Jordt SE, Nikai T, et al. TRPA1 mediates the inflammatory actions of environmental irritants and proalgesic agents. Cell. 2006;124(6):1269-1282.

4. Bautista DM, Pellegrino M, Tsunozaki M. TRPA1: a gatekeeper for inflammation. Annu Rev Physiol. 2013;75:181-200.
5. Atoyan R, Shander D, Botchkareva NV. Non-neuronal expression of transient receptor potential type A1 (TRPA1) in human skin. J Invest Dermatol. 2009;129(9):2312-2315.

6. Albarrán L, Lopez JJ, Dionisio N, Smani T, Salido GM, Rosado JA. Transient receptor potential ankyrin-1 (TRPA1) modulates store-operated $\mathrm{Ca}^{2+}$ entry by regulation of STIM1-Orail association. Biochim Biophys Acta. 2013;1833(12):3025-3034.

7. Nozawa K, Kawabata-Shoda E, Doihara H, et al. TRPA1 regulates gastrointestinal motility through serotonin release from enterochromaffin cells. Proc Natl Acad Sci U S A. 2009;106(9):3408-3413.

8. Obata K, Katsura H, Mizushima T, et al. TRPA1 induced in sensory neurons contributes to cold hyperalgesia after inflammation and nerve injury. J Clin Invest. 2005;115(9):2393-2401.

9. Kobayashi K, Fukuoka T, Obata K, et al. Distinct expression of TRPM8, TRPA1, and TRPV1 mRNAs in rat primary afferent neurons with adelta/c-fibers and colocalization with trk receptors. J Comp Neurol. 2005;493(4):596-606.

10. Anand U, Otto WR, Facer P, et al. TRPA1 receptor localisation in the human peripheral nervous system and functional studies in cultured human and rat sensory neurons. Neurosci Lett. 2008;438(2):221-227.

11. da Costa DS, Meotti FC, Andrade EL, Leal PC, Motta EM, Calixto JB. The involvement of the transient receptor potential A1 (TRPA1) in the maintenance of mechanical and cold hyperalgesia in persistent inflammation. Pain. 2010;148(3):431-437.

12. Kim YS, Son JY, Kim TH, et al. Expression of transient receptor potential ankyrin 1 (TRPA1) in the rat trigeminal sensory afferents and spinal dorsal horn. J Comp Neurol. 2010;518(5):687-698.

13. Huang D, Li S, Dhaka A, Story GM, Cao YQ. Expression of the transient receptor potential channels TRPV1, TRPA1 and TRPM8 in mouse trigeminal primary afferent neurons innervating the dura. Mol Pain. 2012;8:66.

14. Horváth Á, Tékus V, Boros M, et al. Transient receptor potential ankyrin 1 (TRPA1) receptor is involved in chronic arthritis: in vivo study using TRPA1-deficient mice. Arthritis Res Ther. 2016;18:6.

15. Petrus M, Peier AM, Bandell M, et al. A role of TRPA1 in mechanical hyperalgesia is revealed by pharmacological inhibition. Mol Pain. 2007;3:40.

16. Eid SR, Crown ED, Moore EL, et al. HC-030031, a TRPA1 selective antagonist, attenuates inflammatory- and neuropathy-induced mechanical hypersensitivity. Mol Pain. 2008;4:48.

17. McGaraughty S, Chu KL, Perner RJ, Didomenico S, Kort ME, Kym PR. TRPA1 modulation of spontaneous and mechanically evoked firing of spinal neurons in uninjured, osteoarthritic, and inflamed rats. $\mathrm{Mol}$ Pain. 2010;6:14.

18. Wei H, Koivisto A, Saarnilehto M, et al. Spinal transient receptor potential ankyrin 1 channel contributes to central pain hypersensitivity in various pathophysiological conditions in the rat. Pain. 2011;152(3):582-591.

19. Wei H, Karimaa M, Korjamo T, Koivisto A, Pertovaara A. Transient receptor potential ankyrin 1 ion channel contributes to guarding pain and mechanical hypersensitivity in a rat model of postoperative pain. Anesthesiology. 2012;117(1):137-148.

20. Vermeulen W, De Man JG, De Schepper HU, et al. Role of TRPV1 and TRPA1 in visceral hypersensitivity to colorectal distension during experimental colitis in rats. Eur J Pharmacol. 2013:698(1-3): 404-412.

21. Bonet IJ, Fischer L, Parada CA, Tambeli CH. The role of transient receptor potential A 1 (TRPA1) in the development and maintenance of carrageenan-induced hyperalgesia. Neuropharmacology. 2013;65:206-212.

22. Schwartz ES, La JH, Scheff NN, Davis BM, Albers KM, Gebhart GF. TRPV1 and TRPA1 antagonists prevent the transition of acute to chronic inflammation and pain in chronic pancreatitis. $J$ Neurosci. 2013;33(13):5603-5611.

23. Asgar J, Zhang Y, Saloman JL, Wang S, Chung MK, Ro JY. The role of TRPA1 in muscle pain and mechanical hypersensitivity under inflammatory conditions in rats. Neuroscience. 2015;310:206-215. 
24. Chen J, Joshi SK, DiDomenico S, et al. Selective blockade of TRPA1 channel attenuates pathological pain without altering noxious cold sensation or body temperature regulation. Pain. 2011;152(5):1165-1172.

25. Wei H, Saarnilehto M, Falck L, et al. Spinal transient receptor potential ankyrin 1 channel induces mechanical hypersensitivity, increases cutaneous blood flow, and mediates the pronociceptive action of dynorphin A. J Physiol Pharmacol. 2013;64(3):331-340.

26. Zhang W, Liu Y, Zhao X, Gu X, Ma Z. The effect of intrathecal administration TRPA1 antagonists in a rat model of neuropathic pain. Anesth Analg. 2014;119(1):179-185.

27. Miyakawa T, Terashima Y, Takebayashi T, et al. Transient receptor potential ankyrin 1 in spinal cord dorsal horn is involved in neuropathic pain in nerve root constriction rats. Mol Pain. 2014;10:58.

28. Trevisan G, Benemei S, Materazzi S, et al. TRPA1 mediates trigeminal neuropathic pain in mice downstream of monocytes/macrophages and oxidative stress. Brain. 2016 139(Pt 5):1361-1377.

29. Zimmermann M. Ethical guidelines for investigations of experimental pain in conscious animals. Pain. 1983;16(2):109-110.

30. Wheeler-Aceto H, Cowan A. Standardization of the rat paw formalin test for the evaluation of analgesics. Psychopharmacology (Berl). 1991;104(1):35-44.

31. Fu KY, Light AR, Maixner W. Relationship between nociceptor activity, peripheral edema, spinal microglial activation and long-term hyperalgesia induced by formalin. Neuroscience. 2000;101(4):1127-1135.

32. Ambriz-Tututi M, Rocha-González HI, Castañeda-Corral G, et al. Role of opioid receptors in the reduction of formalin-induced secondary allodynia and hyperalgesia in rats. Eur J Pharmacol. 2009;619(1-3):25-32.

33. Leem JW, Willis WD, Weller SC, Chung JM. Differential activation and classification of cutaneous afferents in the rat. $J$ Neurophysiol. 1993;70(6):2411-2424.

34. Martínez-Rojas VA, Barragán-Iglesias P, Rocha-González HI, Murbartián J, Granados-Soto V. Role of TRPV1 and ASIC3 in formalin-induced secondary allodynia and hyperlagesia. Pharmacol Reports. 2014;66(6):964-971.

35. McNamara CR, Mandel-Brehm J, Bautista DM, et al. TRPA1 mediates formalin-induced pain. Proc NatlAcad Sci USA. 2007;104(33):13525-13530.

36. Ambriz-Tututi M, Cruz SL, Urquiza-Marín H, Granados-Soto V. Formalininduced long-term secondary allodynia and hyperalgesia are maintained by descending facilitation. Pharmacol Biochem Behav. 2011;98(3):417-424.

37. Fernandes ES, Russell FA, Spina D, et al. A distinct role for transient receptor potential ankyrin 1 , in addition to transient receptor potential vanilloid 1 , in tumor necrosis factor $\alpha$-induced inflammatory hyperalgesia and Freund's complete adjuvant-induced monarthritis. Arthritis Rheum. 2011:63(3):819-829.

38. Mueller-Tribbensee SM, Karna M, Khalil M, Neurath MF, Reeh PW, Engel MA. Differential contribution of TRPA1, TRPV4 and TRPM8 to colonic nociception in mice. PLoS One. 2015;10(7): 0128242.

39. Dunham JP, Kelly S, Donaldson LF. Inflammation reduces mechanical thresholds in a population of transient receptor potential channel A1-expressing nociceptors in the rat. Eur J Neurosci. 2008;27(12):3151-3160.

40. Granados-Soto V, Alonso-López R, Asomoza-Espinosa R, Rufino MO, Gomes-Lopes LD, Ferreira SH. Participation of COX, IL-1 beta and TNF alpha in formalin-induced inflammatory pain. Proc West Pharmacol Soc. 2001;44:15-17.

41. Parada CA, Tambeli CH, Cunha FQ, Ferreira SH. The major role of peripheral release of histamine and 5-hydroxytryptamine in formalininduced nociception. Neuroscience. 2001;102(4):937-944.

Journal of Pain Research

Publish your work in this journal

The Journal of Pain Research is an international, peer reviewed, open access, online journal that welcomes laboratory and clinical findings in the fields of pain research and the prevention and management of pain. Original research, reviews, symposium reports, hypothesis formation and commentaries are all considered for publication.
42. Nakajima K, Obata H, Ito N, Goto F, Saito S. The nociceptive mechanism of 5-hydroxytryptamine released into the peripheral tissue in acute inflammatory pain in rats. Eur J Pain. 2009;13(5):441-447.

43. Malmberg AB, Yaksh TL. Cyclooxygenase inhibition and the spinal release of prostaglandin $\mathrm{E}_{2}$ and amino acids evoked by paw formalin injection: a microdialysis study in unanesthetized rats. $J$ Neurosci. 1995; 15(4):2768-2776.

44. Wei H, Koivisto A, Pertovaara A. Spinal TRPA1 ion channels contribute to cutaneous neurogenic inflammation in the rat. Neurosci Lett. 2010:479(3):253-256.

45. Watkins LR, Martin D, Ulrich P, Tracey KJ, Maier SF. Evidence for the involvement of spinal cord glia in subcutaneous formalin induced hyperalgesia in the rat. Pain. 1997:71(3):225-235.

46. Fu KY, Tan YH, Sung B, Mao J. Peripheral formalin injection induces unique spinal cord microglial phenotypic changes. Neurosci Lett. 2009;449(3):234-239.

47. Wei F, Dubner R, Zou S, Ren K, Bai G, Wei D, Guo W. Molecular depletion of descending serotonin unmasks its novel facilitatory role in the development of persistent pain. J Neurosci. 2010;30(25):8624-8636.

48. Weller K, Reeh PW, Sauer SK. TRPV1, TRPA1, and CB1 in the isolated vagus nerve - axonal chemosensitivity and control of neuropeptide release. Neuropeptides. 2011;45(6):391-400.

49. Kosugi M, Nakatsuka T, Fujita T, Kuroda Y, Kumamoto E. Activation of TRPA1 channel facilitates excitatory synaptic transmission in substantia gelatinosa neurons of the adult rat spinal cord. $J$ Neurosci. 2007;27(16):4443-4451.

50. Wrigley PJ, Jeong HJ, Vaughan CW. Primary afferents with TRPM8 and TRPA1 profiles target subpopulations of rat superficial dorsal horn neurons. Br J Pharmacol. 2009;157(3):371-380.

51. Steen KH, Steen AE, Kreysel HW, Reeh PW. Inflammatory mediators potentiate pain induced by experimental tissue acidosis. Pain. 1996;66(2-3):163-170.

52. Rocha-González HI, Herrejon-Abreu EB, López-Santillán FJ, GarcíaLópez BE, Murbartián J, Granados-Soto V. Acid increases inflammatory pain in rats: effect of local peripheral ASICs inhibitors. Eur J Pharmacol. 2009;603(1-3):56-61.

53. Castañeda-Corral G, Rocha-González HI, Araiza-Saldaña CI, et al. Blockade of peripheral and spinal $\mathrm{Na}^{+} / \mathrm{H}^{+}$exchanger increases formalininduced long-lasting mechanical allodynia and hyperalgesia in rats. Brain Res. 2012;1475:19-30.

54. Leffler A, Mönter B, Koltzenburg M. The role of the capsaicin receptor TRPV1 and acid-sensing ion channels (ASICS) in proton sensitivity of subpopulations of primary nociceptive neurons in rats and mice. Neuroscience. 2006;139(2):699-709.

55. Frederick J, Buck ME, Matson DJ, Cortright DN. Increased TRPA1, TRPM8, and TRPV2 expression in dorsal root ganglia by nerve injury. Biochem Biophys Res Commun. 2007;358(4):1058-1064.

56. Caspani O, Zurborg S, Labuz D, Heppenstall PA. The contribution of TRPM8 and TRPA1 channels to cold allodynia and neuropathic pain. PLoS One. 2009;4(10):e7383.

57. Ji G, Zhou S, Carlton SM. Intact A delta-fibers up-regulate transient receptor potential A1 and contribute to cold hypersensitivity in neuropathic rats. Neuroscience. 2008;154(3):1054-1066.

58. Yang J, Li Y, Zuo X, Zhen Y, Yu Y, Gao L. Transient receptor potential ankyrin-1 participates in visceral hyperalgesia following experimental colitis. Neurosci Lett. 2008;440(3):237-241.

The manuscript management system is completely online and includes a very quick and fair peer-review system, which is all easy to use. Visit http://www.dovepress.com/testimonials.php to read real quotes from published authors. 